\title{
Elastica and buckling loads of nonlinear elastic tapered cantilever columns
}

\author{
Joon Kyu Lee and Byoung Koo Lee ${ }^{b^{*}}$
}

${ }^{a}$ Department of Civil Engineering, University of Seoul, Seoul, Korea

${ }^{b}$ Department of Civil and Environmental Engineering, Iksan, Jeollabuk-do, Korea

\begin{tabular}{l}
\hline A R T I C L EI N F O \\
\hline Article history: \\
Received 26 June, 2017 \\
Accepted 1 November 2017 \\
Available online \\
1 November 2017 \\
\hline Keywords: \\
Elastica \\
Buckling load \\
Tapered column \\
Ludwick type material \\
\hline
\end{tabular}
A B S T R A C T

\begin{abstract}
This paper deals with the elastica and buckling loads of the nonlinear elastic tapered cantilever columns subjected to an axial load at the free end. The column cross section is rectangular, where the width and depth vary linearly with the column axis. The column material is nonlinear elastic, which obeys the Ludwick's constitutive law. The ordinary differential equations governing the elastica of buckled column are derived and solved numerically for computing the elastica and buckling loads. Parametric studies for the annealed copper, N.P. 8 aluminum alloy and steel columns are conducted.
\end{abstract}

C 2018 by the authors; licensee Growing Science, Canada

\section{Introduction}

Elastica problems dealt with the large deflection beam theory are one of the greatest interest themes in the field of structural engineering. Since the first study of the elastica was published by Euler (1774), in which large deformed shapes of a slender rod were studied, such elastica problems have been still discussed to the present day. Much reference, particularly on the cantilever beams/columns, and their citations include the mathematical models and the significant historical literature on this subject. Concerning the large deformation beam theory, typical works related to this study should be classified into two column materials: the linear and nonlinear elastic materials. Firstly, very much works related to the elastica of the linear elastic materials had been published in the past few decades. For typical examples, various effects on the elastica responses had been extensively investigated, such as solution methods (Berkey \& Freedman, 1978; Panayotounakos \& Theocaris, 1988; Lee \& Lee, 2017); loading conditions (Berkey \& Freedman, 1978; Nageswara \& Rao, 1990; Lee \& Oh, 2000; Borboni \& Santis, 2014); variable cross sections (Nageswara \& Rao, 1990; Lee \& Oh, 2000; Lee \& Lee, 2017); optimal shapes (Berkey \& Freedman, 1978; Lee \& Lee, 2017); and secondary effects such as shear deformation (Nageswara \& Rao, 1990; Lee \& Lee, 2017).

* Corresponding author.

E-mail addresses: bkleest@wku.ac.kr (B. K. Lee) 
Secondly, works dealing with the elastica of the nonlinear elastic materials which obey the Ludwick type constitutive law directly related to the present study are very limited. For elastica problems of the nonlinear elastic beams, several works are shortly introduced: Lewis and Monasa $(1981,1982)$ solved the large deflections of the prismatic beams subjected to the point load and tip couple, respectively; Lee (2002) studied the large deformed prismatic beams with both the tip point load and uniform load; Jung and Kang (2005) studied the large deflections of cantilever beams with the nonlinear elastic materials; Eren (2008) solved the large deflections of the rectangular combined loaded beams by means of different arc length assumptions; and Brojan et al. (2009) investigated the large deflections of nonprismatic/tapered beams with the tip couple.

For elastica problems of the nonlinear elastic column, very limited number of works had been published in the past few decades. Typical works are shortly reviewed: Kounadis and Mallis (1978) presented the post-buckling response of a simply supported, axially compressed, uniform bar of nonlinearly elastic material, in which the bar axis shortening was taken into account; Haslach (1985) analyzed the post-buckling behavior of the column made of a material with cubic constitutive equation to discover the post-buckling load deflection relationship; Lee (2001) investigated the post-buckling tip responses of uniform column under a combined load consisting of a uniformly distributed axial load and concentrated load at the free end; Jung and Kang (2005) studied the large deflections of cantilever columns with the modified Ludwick nonlinear elastic materials; Brojan et al. $(2007,2009)$ presented the buckling stability characteristics of nonlinear elastic column, depending upon the values of different material parameters; and Saetiew and Chucheepsakul (2012) studied the post-buckling behavior of the linearly tapered and simply supported column made of the nonlinear elastic materials.

This paper aims to investigate the elastica and buckling loads of the nonlinear elastic tapered cantilever columns subjected to an axial compressive load. The column cross section is solid rectangular, whose width and depth are linearly varied, respectively, along the column axis. The nonlinear column material obeys the Ludwick type constitutive law. Analyses of the elastica are based on the large deflection beam theory, which deals with the geometric nonlinear differential equations that relate the deflection to the load. Following assumptions are inherent: the column material is nonlinear elastic; the cross section is rectangular; the neutral axis for bending is incompressible; the plane sections remain plane; the shear deformation is ignored; and the axial load direction is vertical.

This study begins with defining both the width and depth of the tapered rectangular column. It derives differential equations which govern the elastica of the buckled column, and then solves these equations numerically for calculating the elastica and buckling loads using the Runge-Kutta and Regula-Falsi methods. The buckling loads and tip responses of the elastica obtained from this study are compared to those in the open literature for the validation purpose. This study extensively discusses numerical results of the elastica and buckling loads for the annealed copper column, and finally ends with comparisons the buckling load elastica and extreme stresses for three different column materials of the annealed copper, N.P. 8 aluminum alloy and steel. Nowhere in the open literature were found solutions for the buckling analysis of cantilever columns considered both the nonlinear elastic (Ludwick type material) and taper effects.

\section{Mathematical modelling}

Shown in Fig. 1 is the geometry of the tapered cantilever column with the length $l$, where the bottom end $c$ is clamped and top end $f$ is free. The column cross section is solid rectangular, whose width and depth vary linearly in $z$ and $y$ directions, respectively, with the axial length $s$ measured from the clamped end. The width and depth at any $s$ are represented as $W$ and $D$, respectively. Those of the clamped end $c(s=0)$ are $W_{c}$ and $D_{c}$, and those of the free end $f(s=l)$ are $W_{f}$ and $D_{f}$, respectively. In addition, note that $z$ axis is the bending axis. 
For functionally expressing $W$ and $D$ with $s$, following two nondimensional section ratios are defined as

$$
\begin{aligned}
& r_{w}=\frac{W_{f}}{W_{c}} \\
& r_{d}=\frac{D_{f}}{D_{c}}
\end{aligned}
$$

Considering $r_{w}$ and $r_{d}$ introduced above, $W$ and $D$ at any $s$ are expressed as a function of $s$, or

$$
\begin{aligned}
& W=W_{c}\left[\left(r_{w}-1\right) \frac{s}{l}+1\right]=W_{c}\left(\alpha_{w} \frac{s}{l}+1\right) \\
& D=D_{c}\left[\left(r_{d}-1\right) \frac{s}{l}+1\right]=D_{c}\left(\alpha_{d} \frac{s}{l}+1\right)
\end{aligned}
$$

where, $\alpha_{w}=r_{w}-1$ and $\alpha_{d}=r_{d}-1$.

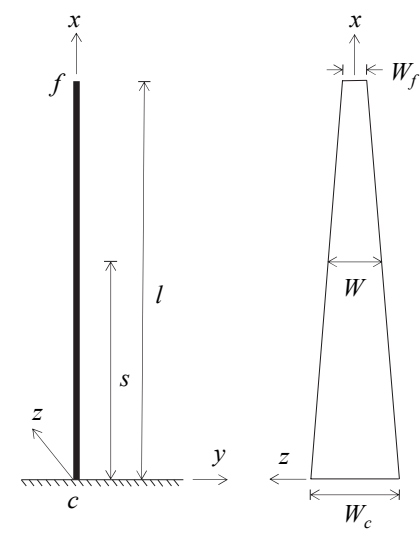

Fig. 1. Geometry of tapered cantilever column

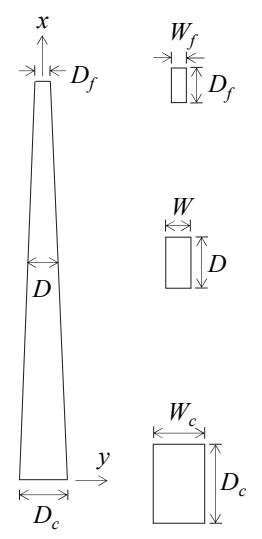

Symbols and loadings for the cantilever column with length $l$ are defined in Fig. 2, which is subjected to an axial load $P$. The pre-buckled column, illustrated as a dashed line, is still straight, since $P$ is less than the buckling load $B(P<B)$. However, once $P$ becomes to be greater than $B(P>B)$, the column buckles and forms the elastica, an exact shape of the buckled axis, depicted as a solid curve. The elastica is defined in the rectangular coordinates $(x, y)$. The buckled arc length $s$ at $(x, y)$ is measured from the clamped end as previously depicted in Fig. 1. For the pre-buckled column, the axis coincides with the vertical $x$-axis, whereas for the post-buckled column, the axis no longer aligns with the $x$-axis. After buckling the column, the material point marked by $\circ$ on the straight axis moves to the material point marked by $\bullet$ on the post-buckled axis. The arc length $s$ at the free end maintains the length $l$ because of the assumption of incompressibility. The angle rotation of the elastica is depicted as $\theta$. The axial force, shear force, and bending moment on the elastica are depicted as $A, Q$ and $M$, respectively, where their respective positive sign conventions are presented in Fig. 2. At the free end, the horizontal and vertical deflections, and angle rotation are illustrated as $\Delta_{h}$ and $\Delta_{v}$, and $\theta_{f}$, respectively. From the stsatcs, stress resultants of $A, Q$ and $M$ at any $s$ on the elastica are expressed as

$$
\begin{aligned}
& A=P \cos \theta \\
& Q=P \sin \theta \\
& M=P\left(\Delta_{h}-y\right)
\end{aligned}
$$

If a cross section is defined and a stress-strain curve of the material is prescribed, an equation connecting curvature $\kappa$ and moment $M$ can be established. In this paper, the column is made of a nonlinear material where the stress-strain curve is represented by the Ludwick relation (Lee, 2001, 2002), that is

$$
\sigma=E|\varepsilon|^{1 / n}
$$


where $\sigma$ and $\varepsilon$ represent the stress and strain, and the constants $E$ and $n$ represent the mechanical properties of the material, respectively. Note that the sign of $\sigma$ in Eq. (8) follows that of $\varepsilon$.

The bending moment-curvature relationship for the rectangular cross section with $W$ and $D$ of the Ludwick type nonlinear elastic material can be written as (Lee, 2002),

$$
M=E \frac{W D^{2+1 / n}}{(2+1 / n) 2^{1+1 / n}} \kappa^{1 / n}=E \frac{W D^{2+1 / n}}{(2+1 / n) 2^{1+1 / n}}\left(\frac{d \theta}{d s}\right)^{1 / n}
$$

in which the term of $d \theta / d s$ is selected rather than that of curvature $\kappa(=d \theta / d s)$ for the sake of convenience in developing the elastica theories hereafter.

Substituting Eq. (7) into Eq. (9) together with Eqs. (3) and Eq. (4) and rearranging gives

$$
\frac{d \theta}{d s}=\left[\frac{(2+1 / n) 2^{1+1 / n} P}{E W_{c} D_{c}^{2+1 / n}} \frac{\Delta_{h}-y}{\left(\alpha_{w} \frac{s}{l}+1\right)\left(\alpha_{d} \frac{s}{l}+1\right)^{2+1 / n}}\right]^{n} ; 0 \leq s \leq l
$$

In subsequential process, two differential equations are obtained from the large deflection beam theory:

$$
\begin{aligned}
& \frac{d x}{d s}=\cos \theta \\
& \frac{d y}{d s}=\sin \theta
\end{aligned}
$$

Now consider the boundary conditions of the column. At the clamped end $(s=0)$, no deflections occur, or

$$
\begin{aligned}
& x=0 \\
& y=0 \\
& \theta=0
\end{aligned}
$$

Since $\Delta_{h}$ in the differential equation, eq. (10), is unknown, differential equations, eqs. (10)-(12), as an initial value problem, cannot be solved. In order to determine the unknown $\Delta_{h}$, the boundary condition at the free end $(s=l)$ is considered, as a boundary value problem. At the free end, the horizontal deflection $\Delta_{h}$ clearly equals the coordinate $y$, or

$$
\Delta_{h}-y=0
$$

To facilitate numerical studies and to obtain the most general results for this class of the problems, the loadings, geometric parameters, and governing equations with their boundary conditions are cast in the following nondimensional forms. Firstly, the axial load and buckling load parameters are defined as

$$
\begin{aligned}
& p=\frac{P l^{2}}{E I_{c}}, \\
& b=\frac{B l^{2}}{E I_{c}},
\end{aligned}
$$

where, $I_{c}=W_{c} D_{c}^{3} / 12$.

The stress resultants of $A, Q$ and $M$ are normalized as

$$
\begin{aligned}
a & =\frac{A l^{2}}{E I_{c}} \\
q & =\frac{Q l^{2}}{E I_{c}}
\end{aligned}
$$


$m=\frac{M l}{E I_{C}}$

The width and depth at the clamped end are normalized by the length $l$ as

$w_{c}=\frac{W_{c}}{l}$

$d_{c}=\frac{D_{c}}{l}$

The arc length $s$ and coordinates $(x, y)$ are normalized by the length $l$, or

$\begin{aligned} \lambda & =\frac{s}{l} \\ \xi & =\frac{x}{l} \\ \eta & =\frac{y}{l}\end{aligned}$

The tip deflections of $\Delta_{h}$ and $\Delta_{v}$ at the free end are also normalized by the length $l$, and the angle parameter $\varphi_{f}$ against the tip rotation $\theta_{f}$ is defined as

$$
\begin{gathered}
\delta_{h}=\frac{\Delta_{h}}{l} \\
\delta_{v}=\frac{\Delta_{v}}{l} \\
\varphi_{f}=\frac{\theta_{f}}{\pi}
\end{gathered}
$$

where it is particularly noted that the elastica has negative curvature when $\varphi_{f}$ is larger than 0.5 , i.e., $\varphi_{f}>0.5$.

Combining the differential equations of Eqs. (10-12) and the nondimensional parameters of Eqs. (1727) yields

$$
\begin{aligned}
& \frac{d \theta}{d \lambda}=\left[\frac{(2+1 / n) 2^{1+1 / n} p}{12 d_{c}^{1 / n-1}} \frac{\delta_{h}-\eta}{\left(\alpha_{w} \lambda+1\right)\left(\alpha_{d} \lambda+1\right)^{2+1 / n}}\right]^{n} ; 0 \leq \lambda \leq 1 \\
& \frac{d \xi}{d \lambda}=\cos \theta \\
& \frac{d \eta}{d \lambda}=\sin \theta
\end{aligned}
$$

Eqs. (30-32) derived above are the totally third order nondimensional differential equations which govern the buckled shape of the nonlinear elastic tapered cantilever column.

The boundary conditions of the clamped end $(\lambda=0)$, Eqs. (13-15), become

$\xi=0$

$\eta=0$

$\theta=0$

The boundary condition of the free end $(\lambda=1)$, Eq. (16), becomes

$$
\delta_{h}-\eta=0
$$

\section{Numerical methods}

Based on above analyses, two FORTRAN computer programs were written to calculate the elastica and the buckling load. The input column parameters are: the material properties of $E$ and $n$; the length $l$; the column geometry of $W_{c}, D_{c}, W_{f}$ and $D_{f}$; and the axial load $P$. From these parameters given in the dimensional units are transformed into those nondimensional forms: $d_{c}, r_{w}$ and $r_{d}$, and $p$. For integrating differential equations, the Runge-Kutta method (Carnahan \& Luther, 1969) was used and 
for determining the unknown boundary condition $\delta_{h}$ at the free end, the Regula-Falsi method (Carnahan $\&$ Luther, 1969), a solution method of the nonlinear equations, was used. These kinds of numerical methods for the initial and boundary value problems are adopted in the open literature (Lee \& Jeong, 2016; Lee \& Lee, 2017).

Firstly, the numerical processes for solving the elastica are summarized:

1) Set the column parameters of $n, w_{c}, d_{c}, r_{w}, r_{d}$ and $p$.

2) Assume a trial boundary condition $\left(\delta_{h}\right)_{t}$ at the free end $(\lambda=1)$. The first trial is zero.

3 ) Integrate the differential equations, eqs. (30)-(32), subjected to the boundary condition of Eqs. (33$35)$ and assumed $\left(\delta_{h}\right)_{t}$ in above step using the Runge-Kutta method. After complete integrating, the trial solutions of the elastica include $(\xi, \eta, \theta)$ in the range of $0 \leq \lambda \leq 1$.

4) Calculate the trial boundary condition $C$ in Eq. (36) at the free end $(\lambda=1)$ :

$$
C=\left(\delta_{h}\right)_{t}-\eta_{\lambda=1}
$$

If $C=0$, the trial solution computed in step 3 is just the characteristic one. If $|C| \leq E R_{1}$, the trial solution is merely a numerical one. Here, $E R_{1}$ is the first convergence criterion, which is used as $1 \times$ $10^{-8}$ in this study.

5) When the above criterion in step 4 is not satisfied, increment in $\Delta$ to the previous trial $\left(\delta_{h}\right)_{1}$, i.e. new trial $\left(\delta_{h}\right)_{2}=\left(\delta_{h}\right)_{1}+\Delta$, and iterate from step 2 to step 5 .

6) Note the sign of $C_{1} \times C_{2}$, where $C_{1}$ and $C_{2}$ are values of $C$ in the previous and present trials, respectively. If the sign does not change till the trial $\left(\delta_{h}\right)_{2}$ reaches 1 , the given $p$ is less than $b$ and the column is still straight since $\delta_{h}$ cannot exceed unity, and then stop the calculation.

7) If the sign changes, the solution of $\delta_{h}$ lies between $\left(\delta_{h}\right)_{1}$ and $\left(\delta_{h}\right)_{2}$. The advanced trial $\left(\delta_{h}\right)_{3}$ is set by the Regula-Falsi method as:

$$
\left(\delta_{h}\right)_{3}=\frac{\left(\delta_{h}\right)_{1}\left|C_{2}\right|+\left(\delta_{h}\right)_{2}\left|C_{1}\right|}{\left|C_{1}\right|+\left|C_{2}\right|}
$$

8) Once the numerical process is entered into the region of the Regula-Falsi method, execute continuously step 7 until the following criterion is satisfied:

$$
\left|\frac{\left(\delta_{h}\right)_{1}-\left(\delta_{h}\right)_{3}}{\left(\delta_{h}\right)_{3}}\right| \leq E R_{2}
$$

in which $C_{1} \times C_{3}<0$ and $E R_{2}$, second convergence criterion, is used as $1 \times 10^{-5}$ in this study.

If two convergence criteria are satisfied, print the computed elastica of $(\xi, \eta, \theta)$ including tip responses of $\left(\delta_{h}, \delta_{v}, \varphi_{f}\right)$ and stop calculations.

Typical example of $C$ versus $\left(\delta_{h}\right)_{t}$ curve in step 4 is presented in Fig. 3 where two solutions of unstable and stable $\delta_{h}$ are represented on the horizontal axis marked as and $\boldsymbol{\square}$, respectively.

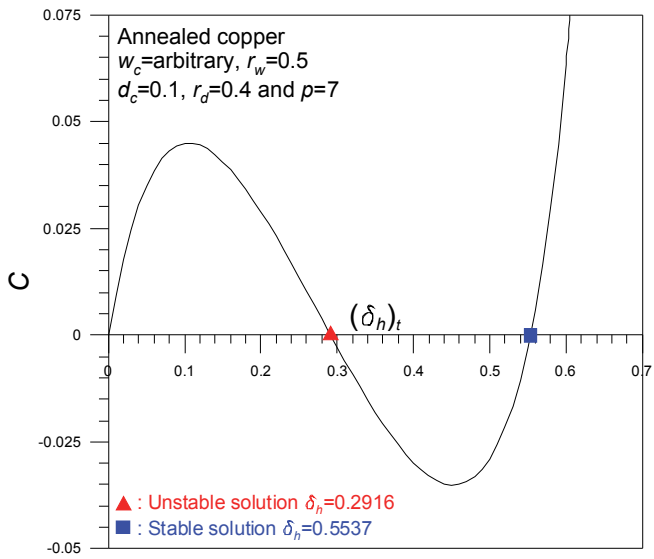

Fig. 3. Example of $C$ versus $\left(\delta_{h}\right)_{t}$ curve 
Secondly, the numerical processes for calculating the buckling load $b$ are summarized: 1) set the column parameters of $n, w_{c}, d_{c}, r_{w}$ and $r_{d}$ naturally except $p$; and then 2) find the minimal $p(=b)$ whose sign of $C_{1} \times C_{2}$ does change during executing from above step 2 to step 6 with the given convergence criteria under which $p(=b)$ is obtained with five significant figures is adopted. All of the computational results of this study are successfully carried out on a desktop computer.

\section{Numerical results}

To conduct the numerical studies on the elastica and the buckling loads, the annealed copper column is typically considered, whose mechanical properties are $E=458.5 \mathrm{MPa}$ and $n=2.160$ as a Ludwick type nonlinear elastic materials

All of the computations were carried out on a desktop personal computer with graphics support. For the numerical results presented herein, a step size of $\Delta \lambda=1 / 50$ in the Runge-Kutta scheme was found to give convergence for $\delta_{h}$ at the free end to within four significant figures.

In the first series of studies, the numerical methods discussed above were used to calculate the buckling loads and tip responses for the steel material of $n=1$, and the results are compared to those available in Lee and Lee (2017) and Lee (2001) are considered. These comparisons are summarized in Table 1 where the column parameters used in comparisons are presented. Table 1 shows that the buckling loads and tip responses of the references and this study are very good agreement for all cases. Since the shear deformation is excluded in this study but included in Lee and Lee (2017), the buckling loads $b$ of this study are slight greater than those of Lee and Lee (2017), whereas tip responses of $\delta_{h}$ and $\delta_{v}$ of this study are slight smaller than those of Lee and Lee (2017). For a while, both works published by Lee (2001) and this study excludes the shear deformation, both results of the buckling loads and tip responses between Lee and Lee (2017) and this study are nearly identical. Such comparisons serve to validate elastica theories and solution methods developed herein.

Table 1. Comparisons ${ }^{*}$ of buckling loads, and tip responses between Refs. [A] (Lee and Lee 2017) and [B] (Lee, 2001), and this study for linear elastic column $(n=1)$ with square cross section $\left(w_{c}=d_{c}\right)$

Buckling load parameter

\begin{tabular}{lcc}
\hline Geometric & \multicolumn{2}{c}{ Buckling load parameter, $b$} \\
\cline { 2 - 3 } parameter & Reference & This study \\
\hline$r_{w}=r_{d}=0.5$ & $0.95643[\mathrm{~A}]$ & 1.0290 \\
$r_{w}=r_{d}=0.58$ & $1.1942[\mathrm{~A}]$ & 1.2528 \\
$r_{w}=r_{d}=1$ & $2.4674[\mathrm{~B}]$ & 2.4674 \\
\hline
\end{tabular}

Tip responses

\begin{tabular}{llllll}
\hline $\begin{array}{l}\text { Load parameter, } \\
p(\geq b)\end{array}$ & Section ratio, & Data source & \multicolumn{3}{c}{ Tip responses } \\
& $r_{w}$ and $r_{d}$ & & $\delta_{h}$ & $\delta_{v}$ & $\varphi_{f}$ \\
\hline 1.250 & $r_{w}=r_{d}=0.5$ & [A] & 0.5105 & 0.2421 & - \\
$(b=1.0290)$ & & This study & 0.5075 & 0.2399 & 0.4143 \\
2.0 & $r_{w}=r_{d}=0.5$ & [A] & 0.6736 & 0.6661 & - \\
$(b=1.0290)$ & & This study & 0.6693 & 0.6619 & 0.7189 \\
$2.4674(=b)$ & $r_{w}=r_{d}=1$ & [B] & 0. & 0. & 0. \\
& & This study & 0. & 0. & 0. \\
$4.64978(b=2.4674)$ & $r_{w}=r_{d}=1$ & [B] & 0.1231 & 0.8032 & 0.6667 \\
& & This study & 0.1233 & 0.8032 & 0.6667 \\
\hline
\end{tabular}

${ }^{*}$ Effect of shear deformation is included in Ref. [A] while excluded in Ref. [B] and this study.

In the second series of studies, the equilibrium paths for the tip responses of $\delta_{h}, \delta_{v}$ and $\varphi_{f}$, elastica of buckled columns $(\xi, \eta)$, buckling loads $b$ and stress resultants of $(a, q, m)$ are evaluated for various combinations of column parameters of $p, w_{c}, d_{c}, r_{w}$ and $r_{d}$. These results for the annealed copper 
column are shown in Figs. 4-7 where all of input column parameters are given in each figure. In addition, the numerical results given in Figs. 4-7 are valid for the arbitrary value of $w_{c}$, i.e., the results are independent on the value of $w_{c}$.
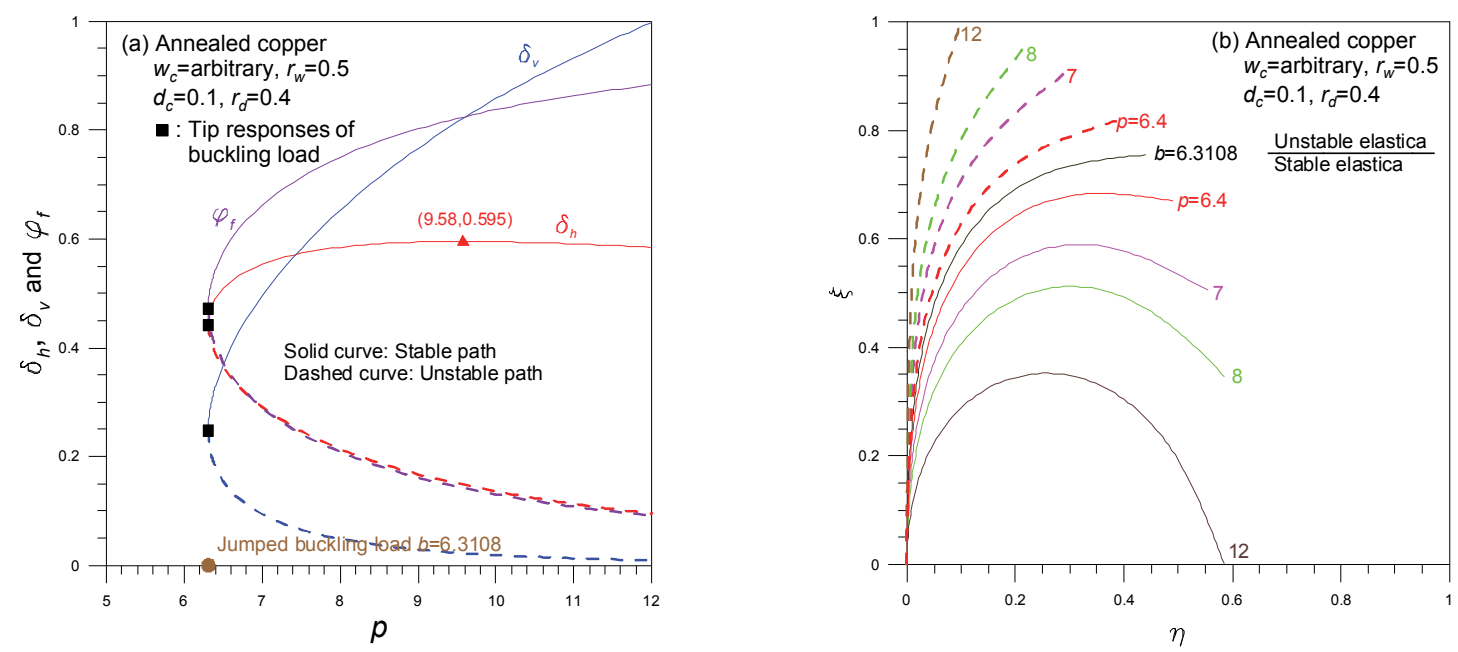

Fig. 4. (a) Equilibrium paths and (b) elastica by varying $p$ for annealed copper column
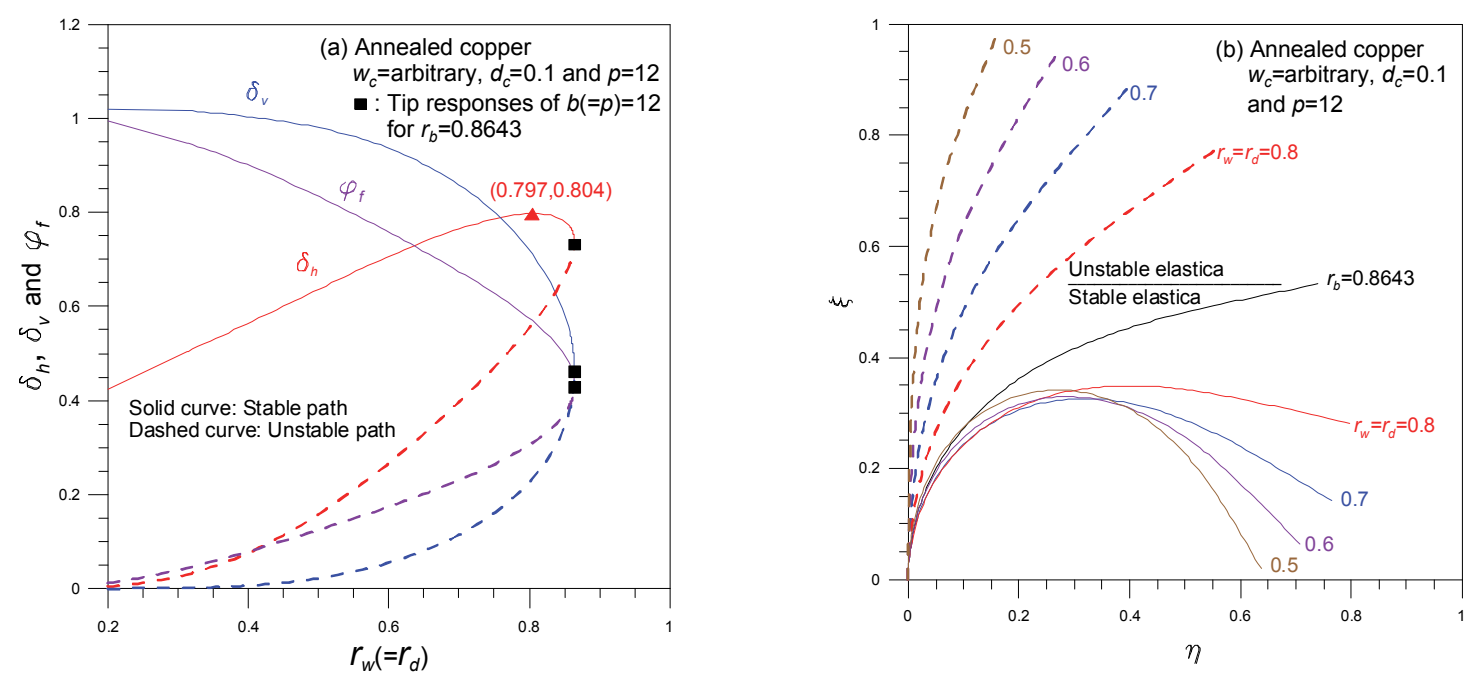

Fig. 5. (a) Equilibrium paths and (b) elastica by varying $r_{w}\left(=r_{d}\right)$ for annealed copper column
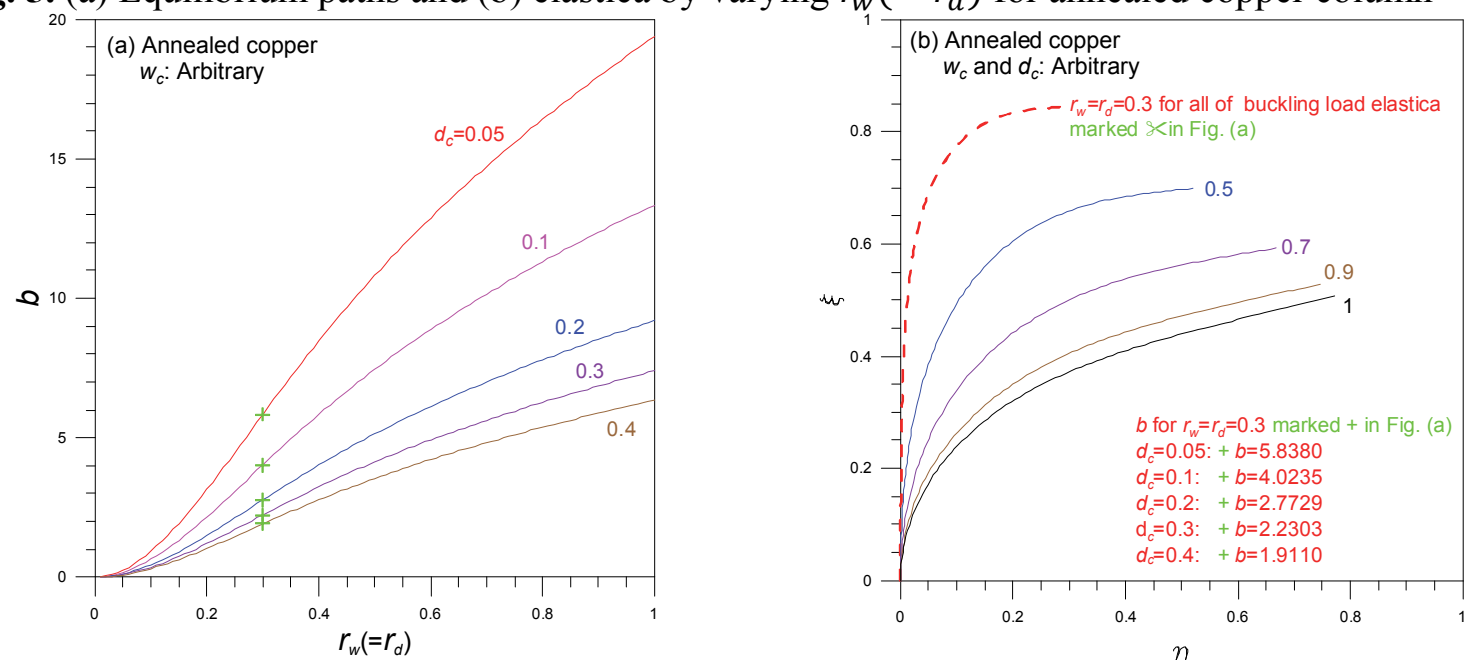

Fig. 6. (a) Buckling loads and (b) buckling load elastica by varying $r_{w}\left(=r_{d}\right)$ for annealed copper column 
Fig. 4 show (a) equilibrium paths and (b) buckled elastica for the annealed copper column, where the column parameters are given. In Fig. 4(a), the column is still straight, which shows the 'zero' equilibrium path, when the load $p$ is less than the buckling load $b$. However, once $p$ reaches $b=$ 6.3108 marked $\bullet$, the column suddenly snaps from the straight column configuration to its buckled elastica which is not adjacent to the straight configuration. As shown in this figure, the buckling tip responses of $\delta_{h}, \delta_{v}$ and $\varphi_{f}$ sudden jump up from zero responses to the respective ones marked $\mathbf{m}$. This kind of buckling is called the snap through buckling, not bifurcation buckling. It is noted that the nonlinear elastic column should be catastrophically collapsed by the snap through buckling. It is expected that the tip responses of $\delta_{h}, \delta_{v}$ and $\varphi_{f}$ mathematically approach 0,2 and 1 , respectively, as $p$ increases. It is seen that the maximum horizontal deflection $\delta_{h(\max )}=0.595$ occurs at $p=9.58$ (see mark $\boldsymbol{\Delta}$ ). After buckling, two equilibrium paths occur as already discussed in Fig. 3 where the one is stable depicted as solid curves and the other is unstable illustrated as dashed curves. The elastica with unstable $\delta_{h}$ has no practical meaning, but only has the mathematical meaning, whereas the elastica with stable $\delta_{h}$ is practical. In Fig. 4(b), the unstable (dashed curve) and stable (solid curve) buckled elastica are shown. The unstable elastica with the smaller $p$ values occur the deeper elastica than those with the larger $p$ ones. This fact is reversing the natural phenomenon so that the unstable elastica is impractical. When the column is subjected to $p=b$ the column catastrophically snaps from the straight configuration to the buckling load elastica with $b=6.3108$. It is noted that the column body subjected to $p=6.3108$ instantaneous travels the trajectories of unstable elastica depicted dashed lines, and finally equilibrates at the buckling elastica. After buckling, the buckled elastica becomes to be deeper as $p$ increases.

Fig. 5 shows (a) equilibrium paths and (b) elastica for annealed copper column with a given $p(=$ 12) by varying $r_{w}\left(=r_{d}\right)$, where the remaining parameters are given. In Fig. 5 (a), it is seen that the column with $r_{w}\left(=r_{d}\right)$ less than $r_{b}=0.8643$ (see mark $\left.\boldsymbol{\square}\right)$ buckles while the column with $r_{w}\left(=r_{d}\right)$ larger than 0.8643 does not buckle and remains the straight state. Thus, the buckling load of the column with $r_{w}\left(=r_{d}\right)=0.8643$ is $b=12$. The tip responses of $\delta_{v}$ and $\varphi_{f}$ decrease as $r_{w}$ increases while that of $\delta_{h}$ increases and reaches peak at $(0.797,0.804)$ (see mark $\boldsymbol{\Delta}$ ), and decreases as $r_{w}$ increases. In Fig. 5(b), the unstable (dashed curve) and stable (solid curve) buckled elastica are shown, where the stable elastica becomes to be deeper as $r_{w}$ decreases.

Fig. 6 shows (a) buckling load parameters $b$ and (b) buckling load elastica by varying $r_{w}\left(=r_{d}\right)$ and $d_{c}$, where the remaining parameters are given. In Fig. 6(a), $b$ of the column increases as $r_{w}\left(=r_{d}\right)$ increase, and $b$ of the column with smaller $d_{c}$ becomes to be larger when the parameter $r_{w}$ is same. For an example, see marks + . In Fig. 8(b), the buckling load elastica of the column with larger $r_{w}\left(=r_{d}\right)$ becomes to be deeper. Particularly, it is very interesting that all of the buckling elastica with the different values of $d_{c}$ are the same if $r_{w}\left(=r_{d}\right)$ of the column is same. For an example, five buckling load elastica, depicted as dashed curve, with $r_{w}\left(=r_{d}\right)=0.3$ are same for the different values of $d_{c}=$ $0.05,0.1,0.2,0.3$ and 0.4 whose corresponding buckling loads $b$ are not same as marked + in Fig. 6(a) and tabulated in Fig. 6(b).

Diagrams of nondimensional stress resultants of $a, q$ and $m$ for the buckled column $(p=7>b=$ 6.3108) are shown in Fig. 7 where the column parameters are presented. From these diagrams, each maximum stress resultant of $a, q$ and $m$, respectively, can be determined, which is absolutely useful for the column design. Particularly, it is seen that the tensile axial force $a$ with negative sign can occur along the column axis in the region of $\varphi_{f}>0.5$. See its corresponding buckling elastica with $p=7$ shown in Fig. 4(b). 


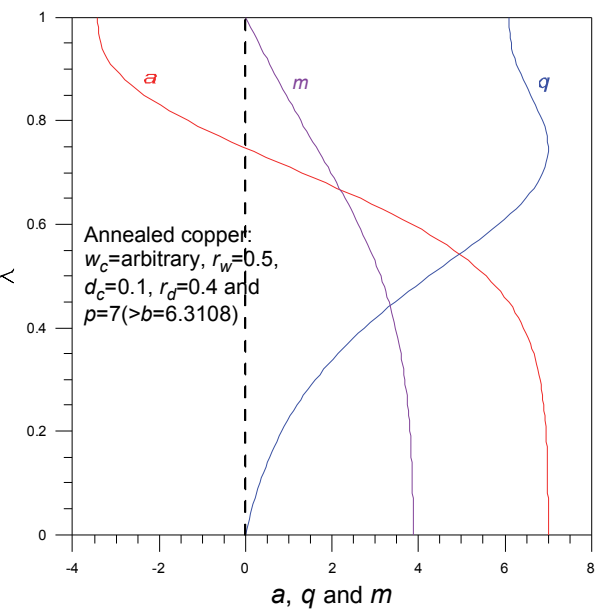

Fig. 7. Diagrams of stress resultants for annealed copper column

In the third series of studies, the buckling analyses are carried out for three different column materials, annealed copper, N.P. 8 aluminum alloy and steel, for the comparison purpose of the buckling behaviors. The mechanical properties of these materials, $E$ and $n$, are as follows.

Annealed copper: $E=458.5 \mathrm{MPa}$ and $n=2.160$;

N.P. 8 aluminum alloy: $E=455.8 \mathrm{MPa}$ and $n=4.785$; and

Steel: $E=207 \mathrm{GPa}$ and $n=1.0$

It is particularly noted that the elastica theories discussed herein are valid not only for the nonlinear elastic materials such as annealed copper and aluminum alloy but also for the linear elastic ones like steel (Lee, 2002).

Fig. 8 shows equilibrium paths against $\varphi_{f}$ by three different column materials. It is seen that $\varphi$ of stable paths, depicted solid curve, increases as $p$ increases for all materials. The buckling load parameters $b$ of the three column materials are the horizontal coordinates $p$ marked $\mathbf{m}$, respectively. The equilibrium path of the steel column bifurcates at the coordinate of the buckling load $b$, while those of annealed copper and aluminum alloy columns jump up at the respective $b$ coordinate from zero to the respective $\varphi_{f}$ coordinate marked $\mathbf{m}$. The dashed curves are unstable equilibrium paths whose responses are always smaller than those of stable paths.

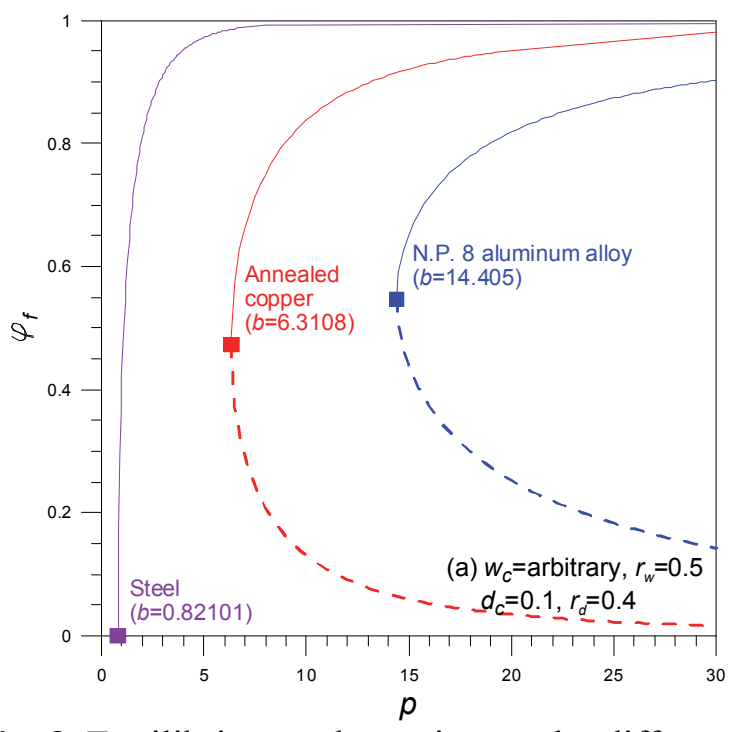

Fig. 8. Equilibrium paths against $\varphi_{f}$ by different column materials

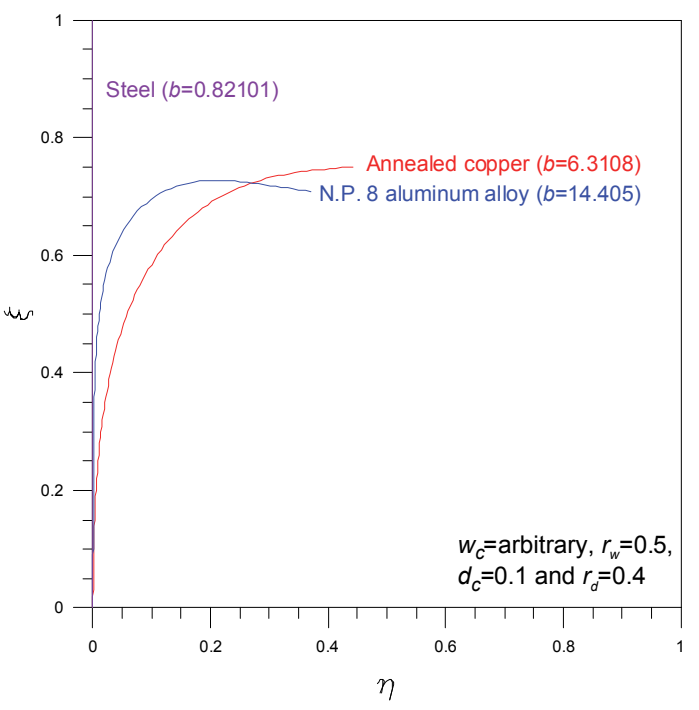

Fig. 9. Buckling load elastica by different column materials 
Fig. 9 shows the buckling load elastica by different column materials, in which the geometrical column parameters are given. The elastica of steel is still straight since the buckling load is the bifurcation one as shown in Fig. 8. On the other hand, the elastica, nevertheless the column are being just after buckling, of the annealed copper and aluminum alloy catastrophically occur the large deformed shapes since these buckling loads are the jumped buckling ones as shown in Fig. 8. The buckling behaviors of these column materials are given in Table 2 .

Table 2. Comparison ${ }^{*}$ of buckling load parameters, and tip responses by column materials

\begin{tabular}{llccc}
\hline Column material & $\begin{array}{l}\text { Buckling load } \\
\text { parameter, } b\end{array}$ & \multicolumn{3}{c}{ Tip responses } \\
\cline { 2 - 5 } & 6.3108 & $\delta_{h}$ & $\delta_{v}$ & $\varphi_{f}$ \\
\hline Annealed copper & 14.405 & 0.4440 & 0.2510 & 0.4735 \\
N.P. 8 aluminum alloy & 0.82101 & 0.3713 & 0.2948 & 0.5469 \\
Steel & ${ }^{*}$ Geometric parameters: $w_{c}=$ arbitrary, $r_{w}=0.5, d_{c}=0.1$ and $r_{d}=0.4$ & & 0. & 0. \\
\hline
\end{tabular}

For steel column, the tip responses are zero, implying the column is still straight. However, the tip responses of copper and aluminum show the very large deformations. In addition, for the aluminum column $\varphi_{f}(=0.5469)$ is greater than $\varphi_{f}=0.5$, implying the curvature $\kappa$ of the buckling elastica becomes negative, and consequently the axial force subjected to the column becomes to be the tension force rather than the compressive force.

\section{Concluding remarks}

This paper studies the elastica and buckling loads of the nonlinear elastic tapered cantilever column subjected to an axial compressive load. The column material obeys the Ludwick type constitutive law. The column cross section is solid rectangular, whose width and depth linearly vary along the column axis. Analysis of the elastica of the buckled column is conducted based on the large deflection beam theory. This analysis derives the totally third order differential equations which govern the buckled elastica, and numerically solves to calculate the buckling loads and elastica. All of the computational results of the elastica and buckling loads are successfully computed on a desktop computer without any difficulties. The buckling loads and tip responses of this study are compared to those available in the open literature for the validation purpose. The numerical results of the buckling loads and elastica responses are extensively discussed. It should be noted that this study considers only rectangular sections and requires further study to extend other types of sections other than rectangular ones, such as the triangle, pentagon, hexigon, circular, etc.

\section{References}

Berkey, D. D., \& Freedman, M. I. (1978). A perturbation method applied to the buckling of a compressed elastica. Journal of Computational and Applied Mathematics, 4(3), 213-221.

Borboni, A., \& De Santis, D. (2014). Large deflection of a non-linear, elastic, asymmetric Ludwick cantilever beam subjected to horizontal force, vertical force and bending torque at the free end. Meccanica, 49(6), 1327-1336.

Brojan, M., Puksic, A., \& Kosel, F. (2007). Buckling and post-buckling of a nonlinearly elastic column. ZAMM-Journal of Applied Mathematics and Mechanics/Zeitschrift für Angewandte Mathematik und Mechanik, 87(7), 518-527.

Brojan, M., Videnic, T., \& Kosel, F. (2009). Large deflections of nonlinearly elastic non-prismatic cantilever beams made from materials obeying the generalized Ludwick constitutive law. Meccanica, 44(6), 733-739.

Brojan, M., Sitar, M., \& Kosel, F. (2012). On static stability of nonlinearly elastic Euler's columns obeying the modified Ludwick's law. International Journal of Structural Stability and Dynamics, 12(06), 1250077.

Carnahan, B., Luther, H. A., \& Wilkes, J. O. (1969). Applied Numerical. Methods, 405-415. 
Eren, I. (2008). Determining large deflections in rectangular combined loaded cantilever beams made of non-linear Ludwick type material by means of different arc length assumptions. Sadhana, 33(1), 45-55.

Euler, L. (1774). Methodus inveniendi lineas curvas maxima minimive proprietate gaudentes, Additamentum I, De Curtis Elasticis, Lausanne and Geneva.

Haslach, H. W. (1985). Post-buckling behavior of columns with non-linear constitutive equations. International journal of non-linear mechanics, 20(1), 53-67.

Jung, J. H., \& Kang, T. J. (2005). Large deflection analysis of fibers with nonlinear elastic properties. Textile research journal, 75(10), 715-723.

Kounadis, A. N., \& Mallis, J. G. (1987). Elastica type buckling analysis of bars from non-linearly elastic material. International journal of non-linear mechanics, 22(2), 99-107.

Lee, B. K., \& Oh, S. J. (2000). Elastica and buckling load of simple tapered columns with constant volume. International Journal of Solids and Structures, 37(18), 2507-2518.

Lee, J. K., \& Lee, B. K. (2016). Large Deflections and Buckling Loads of Cantilever Columns with Constant Volume. International Journal of Structural Stability and Dynamics, 1750091.

Lee, J.K., \& Jeong, S. (2016). Flexural and torsional free vibrations of horizontally curved beams on Pasternak foundations. Applied Mathematical Modelling, 40(3), 2242-2256.

Lee, K. (2001). Post-buckling of uniform cantilever column under a combined load. International Journal of Non-Linear Mechanics, 36(5), 813-816.

Lee, K. (2002). Large deflections of cantilever beams of non-linear elastic material under a combined loading. International Journal of Non-Linear Mechanics, 37(3), 439-443.

Lewis, G., \& Monasa, F. (1981). Large deflections of cantilever beams of nonlinear materials. Computers \& Structures, 14(5-6), 357-360.

Lewis, G., \& Monasa, F. (1982). Large deflections of cantilever beams of non-linear materials of the Ludwick type subjected to an end moment. International Journal of Non-Linear Mechanics, 17(1), 1-6.

Rao, B. N., \& Rao, G. V. (1990). Stability of tapered cantilever columns subjected to a tip-concentrated follower force with or without damping. Computers \& Structures, 37(3), 333-342.

Panayotounakos, D. E., \& Theocaris, P. S. (1988). Analytic solutions for nonlinear differential equatio ns describing the elastica of straight bars: theory. Journal of the Franklin Institute, 325(5), 621-63.

Saetiew, W., \& Chucheepsakul, S. (2012). Post-buckling of linearly tapered column made of nonlinear elastic materials obeying the generalized Ludwick constitutive law. International Journal of Mechanical Sciences, 65(1), 83-96.

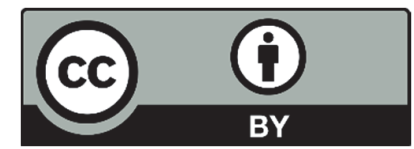

(C) 2017 by the authors; licensee Growing Science, Canada. This is an open access article distributed under the terms and conditions of the Creative Commons Attribution (CC-BY) license (http://creativecommons.org/licenses/by/4.0/). 